\title{
Criminologie
}

\section{Enjeux de la peine et efficacité socio-juridique, le cas du braconnage}

\section{Ruth Murbach}

Volume 20, numéro 2, 1987

La détermination de la sentence

URI : https://id.erudit.org/iderudit/017253ar

DOI : https://doi.org/10.7202/017253ar

Aller au sommaire du numéro

Éditeur(s)

Les Presses de l'Université de Montréal

ISSN

0316-0041 (imprimé)

1492-1367 (numérique)

Découvrir la revue

Citer cette note

Murbach, R. (1987). Enjeux de la peine et efficacité socio-juridique, le cas du braconnage. Criminologie, 20(2), 89-102. https://doi.org/10.7202/017253ar
Résumé de l'article

This note analyzes the perceptions and proceedings of judges and Crown prosecutors when they face a hardly known, decried and severely punished phenomenon: poaching. The findings of a qualitative research indicate that the means and procedures of game protection have unintended implications. The deterrent effect of mandatory minimum penalties is not obvious, while there is evidence that they burden unduly the administration of justice. 


\section{NOTE DE RECHERCHE: ENJEUX DE LA PEINE ET EFFICACITÉ SOCIO-JURIDIQUE, LE CAS DU BRACONNAGE' \\ Ruth Murbach*}

This note analyzes the perceptions and proceedings of judges and Crown prosecutors when they face a hardly known, decried and severely punished phenomenon: poaching. The findings of a qualitative research indicate that the means and procedures of game protection have unintended implications. The deterrent effect of mandatory minimum penalties is not obvious, while there is evidence that they burden unduly the administration of justice.

Parler du braconnage, c'est aborder un phénomène peu connu dont l'importance accordée dépend de la perception de chacun et de son attitude envers la faune. C'est également s'interroger sur les politiques de conservation de la faune et leur application, en tenant compte qu'il s'agit d'une notion floue bien que présente dans l'esprit des chasseurs, bloc de pression, du public en général et des représentants de l'administration de la justice. Y a-t-il concordance entre la vision qu'ont différents groupes sociaux de la conservation de la faune et les politiques en place? Les acteurs du système judiciaire appelés à faire respecter la volonté du législateur ont-ils objectivement et subjectivement les moyens de le faire? Dans quel contexte se réalise leur prise de décision? Comment se représentent-ils cet univers qui n'est pourtant qu'un sous-système de l'administration de la justice?

Dans une recherche précédente ${ }^{2}$, nous constations qu'en tant qu'objet de recherche, le phênomène braconnage a été traité principalement sous deux angles: les estimations du volume de la récolte illégale, afin de déterminer son influence sur les variations des taux de mortalité de populations de différentes espèces, et les attitudes de divers groupes sociaux envers le braconnage et son contrôle. Nous concluions aussi que les méthodes développées pour estimer le volume de la récolte illégale ne sont pas au point. Toutes présentent des failles, soit sur le plan de la fiabilité, soit de la validité. Quant au portrait socio-économique et aux

* Professeur au Département des sciences juridiques à l'Université du Québec à Montréal (UQAM).

1. Cette recherche a été subventionnée par le ministère du Loisir, de la Chasse et de la Pêche du Québec.

2. J. Dozois, P. Landreville, R. Murbach, le Braconnage au Québec: Propositions méthodologiques, Rapport de recherche, École de criminologie, Université de Montréal, 1986. 
motivations du braconnier, nous soulevions que les méthodes traditionnelles des sciences sociales permettent de les déterminer pour les individus figurant dans les fichiers judiciaires. Échappent à ce tableau les braconniers qui n'ont pas été appréhendés. C'est pourquoi la plupart des recherches qui comparent les caractéristiques socio-économiques du braconnier à celles du chasseur ne montrent que très peu de différences entre les deux groupes.

Devant le constat que le phénomène braconnage est mal connu et peu étudié, plusieurs auteurs déplorent que des décisions importantes, aussi bien d'ordre législatif, qu'administratif ou éducatif doivent donc être prises sans connaissances approfondies qui permettraient de mieux évaluer leur impact sur le phénomène et sur les acteurs impliqués. Un tableau global exigerait de considérer les perceptions de différents groupes d'acteurs, tant du côté des utilisateurs de la ressource, légitimes ou illégitimes, que des représentants de l'appareil judiciaire mandatés pour faire respecter la loi, afin de comprendre le contexte social dans lequel l'application de la loi opère. Une connaissance de la vision des uns et des autres serait fondamentale pour l'évaluation de leur interaction. C'est pourquoi nous avons choisi deux groupes d'acteurs qui occupent des positions complémentaires et importantes: les juges de la Cour des sessions de la paix et les procureurs de la Couronne ${ }^{3}$. Les données officielles étant un reflet de leurs décisions, une meilleure connaissance de leurs perceptions du phénomène, de leurs attitudes envers le braconnage, de leurs pratiques et des contextes qui déterminent finalement leurs décisions aidera à comprendre, au moins partiellement, la dynamique de ce système ouvert ${ }^{4}$.

Nous avons opté pour une approche flexible en fonction de notre objet de recherche, utilisé des sources variées et différents types de données et de méthodes. L'étude précédente nous a permis de mieux saisir les dimensions du phénomène. La préparation de l'enquête s'appuyait par ailleurs sur une étude de l'évolution de la législation et de. la jurisprudence, sur la consultation d'un nombre considérable de dossiers d'enquêtes, aussi bien de causes courantes que de grandes opérations. Plusieurs entretiens avec des responsables tant au ministère de la Justice qu'au ministère du Loisir, de la Chasse et de la Pêche nous ont informés des processus administratifs et du cheminement que fait un

3. Nous remercions Marie Beaulieu qui a réalisé la plupart des entrevues et qui a participé à l'organisation du matériel et à une première analyse.

4. La problématique des droits de chasse et de pêche en milieu autochtone a été exclue de cette étude puisqu'elle relève d'une autre compétence législative. 
dossier d'une enquête spécifique. En plus, lors d'une entrevue avec un journaliste spécialisé dans le domaine de la chasse et de la pêche nous avons pu aborder l'évolution des politiques de conservation de la faune au Québec. D'un autre côté, à partir de quelques statistiques disponibles, nous avons tenté d'évaluer à la fois l'importance quantitative des causes reliées à la faune, selon leur gravité ou par région administrative, et le traitement réservé à ces dossiers. Finalement, le fait d'accompagner des agents de conservation pendant une journée de travail nous a donné une certaine expérience du terrain. Le guide d'entretien à questions ouvertes fut développé à partir de l'ensemble de ces informations.

Notre échantillon se constitue de dix juges et de onze procureurs de la Couronne, choisis en fonction des dix régions administratives du ministère du Loisir, de la Chasse et de la Pêche du Québec; il est varié en termes d'années et de types d'expérience des répondants, et nous y avons inclus des chasseurs et des non-chasseurs. L'analyse de contenu, thématique et sémantique, de ces entrevues semi-structurées est à la base de nos conclusions. Cette enquête qualitative a voulu éclairer une partie du système d'application de la Loi sur la conservation et la mise en valeur de la faune et de ses règlements. Nos conclusions se fondent sur l'analyse des perceptions et pratiques des juges et procureurs de la Couronne que nous avons rencontrés. Elles sont valides en elles-mêmes. Il serait pourtant erroné de généraliser ces constats à l'ensemble des procureurs de la Couronne et des juges de la province ou d'une région spécifique. Ceci sera possible à partir des résultats de l'étude quantitative, complémentaire, que nous proposons de réaliser.

Le phénomène braconnage est mal connu; nous l'avons constaté. D'autres chercheurs en ont fait état et l'ont souligné. Nous savons peu sur son incidence, et les méthodes développées à ce jour n'ont guère fait avancer les connaissances. Mais la notion de braconnage elle-même pose un problème. Une définition claire serait pourtant un premier pas nécessaire si l'on veut renforcer le respect de la législation qui protège la faune. Une vision commune du phénomène braconnage n'existe ni dans le groupe des acteurs de l'appareil judiciaire, mandatés pour faire respecter la loi, ni dans le public. Tantôt considéré comme un "défoulement collectif», le braconnage est aussi bien défini dans un sens large, de manière juridique, que par des dimensions qui rendent cette activité particulièrement grave. Ceux qui distinguent entre infractions techniques et le «vrai» braconnage, y associent la chasse de nuit ou hors saison, l'utilisation de moyens interdits spécialement répugnants, la destruction d'espèces menacées et la prise de gibier ou de poisson en grande quantité pour en faire le commerce. Les représentations que juges et procureurs se 
font du phénomène sont influencées par l'importance qu'ils accordent à la faune, la région dans laquelle ils vivent, leur pratique et préférences professionnelles, leur vie privée, les médias.

Aussi varié que la définition du braconnage est le portrait du braconnier tel que l'ont dessiné les interviewés. Il change dans le temps et dans l'espace, selon les contextes et pratiques de chasse ou de pêche. Généralement un «mordu» de la chasse, le braconnier peut venir de tout milieu socio-économique ou professionnel. Quantitativement plus important parmi ceux qui se font prendre sont les braconniers "par tradition" qui ont été initiés tôt par le père ou d'autres proches parents. Pour eux, le braconnnage fait partie des coutumes locales; la tradition les justifie. Comme ils contestaient jadis les clubs privés, en chassant sur leurs territoires, ils contestent maintenant une réglementation lourde, qu'ils prétendent pourtant ignorer. Le plus souvent, ils travaillent dans l'usine ou vivent des prestations du bien-être social. İls sont peu instruits et pauvres. Leur entourage immédiat les appuie, participe même, jusqu'au moment où un parent les dénonce.

Un autre type de braconnier vient plutôt d'une autre région, voire de la ville, et se défoule une fois par année, loin de tout en forêt en compagnie de quelques amis et de beaucoup d'alcool. Là, il se croit tout permis, et il n'hésite pas à aider un peu la chance, soit en chassant avant l'ouverture de la chasse, soit en utilisant des moyens illicites. Il vient d'un milieu socio-économique plutôt aisé, occupe un bon emploi ou travaille comme professionnel à son propre compte.

Mais les attitudes variées des juges et des procureurs de la Couronne se sont manifestées plus clairement dans leur jugement moral du braconnier. S'il y en a qui estiment le phénomène peu important et qui considèrent le braconnier comme quelqu'un qui triche un peu, comme cela peut arriver à tout le monde, d'autres le voient comme un volontariste entêté. Quelques-uns le blâment pour son comportement antisportif et ne lui pardonnent pas d'enfreindre le code d'éthique de la chasse. Dans l'attitude extrême, finalement, une extrapolation de la cruauté envers les animaux transforme le braconnier en individu dangereux pour la société qui fonctionne selon un schèma de pensée déficient et qui n'hésiterait pas à s'attaquer aux humains. Les attitudes varient donc de la compréhension jusqu'au blâme extrême.

Les motivations du braconnier, telles que perçues par les juges et les procureurs de la Couronne, coïncident avec le tableau que les recherches surtout américaines offrent. Dépendant de la définition du braconnage, la typologie se limite aux mêmes catégories de motifs: la consommation 
personnelle, par besoin ou par goût, le profit que l'on peut tirer de la vente, la "fièvre" de la chasse ou les circonstances, i.e. l'occasion qui se présente. Les motifs perçus semblent être liés au degré d'acceptation ou de rejet du phénomène par chaque interviewé. L'enquête par questionnaire permettrait de vérifier cette hypothèse.

Les statistiques du ministère du Loisir, de la Chasse et de la Pêche montrent que le nombre de causes est resté relativement stable depuis les dernières trois années $(1984: 7657,1985: 7835,1986: 6514)$. Le nombre d'infractions pour lesquelles une plainte a été portée diminue tendenciellement (1984: 9 530, 1985: 9 478, 1986: 5 441). Il serait hasardeux de conclure de ce fait une diminution du phénomène dans la province, le chiffre noir demeurant inconnu. Nous constatons d'importantes variations entre les différentes régions administratives, ainsi qu’à l'intérieur des régions, d'une année à l'autre. Si l'Outaouais fut en tête de liste pour l'année 1983-1984, avec 2044 infractions, le nombre de causes y a baissé depuis ( 19851390 ), et ce sont les régions administratives de Québec (1985:1 674) et de Montréal (1985:1 598) qui ont pris la relève.

La distribution selon les catégories de gravité de l'infraction prouve que la grande majorité des actes incriminés sont dans la catégorie légère (1983 et 1984:84,2\%, 1985: 72,6\%). Les infractions de gravité moyenne sont toujours demeurées les moins nombreuses (1983: 3,8\%, 1984 : $6,8 \%, 1985: 11,7 \%$ ), tandis que dans la catégorie des infractions lourdes, on constate, depuis trois ans, une certaine stabilité (autour de $11 \%$ ). Il est important de souligner le nombre important de causes dans la catégorie des infractions légères, puisque c'est à ces plaintes que les inculpés offrent habituellement des plaidoyers de culpabilité. Les juges et les procureurs de la Couronne y investissent donc peu de temps en termes de procès. Par ailleurs, plusieurs excluent ces infractions considérées comme techniques de leur définition du braconnage.

Les données sur trois types d'infractions lourdes (chasse de nuit, usage d'engins prohibés, vente illégale), tant selon la loi que selon les perceptions de nos interviewés, démontrent que ces cas sont peu nombreux. La gravité perçue ne peut donc être une conséquence du fait que juges et procureurs auraient souvent à en traiter.

Les seules données disponibles sur le traitement réservé aux dossiers, après que le ministère du Loisir, de la Chasse et de la Pêche les ait déclarés complets, proviennent du ministère de la Justice. Ce traitement varie en fonction des lois appliquées dans le domaine de la faune. Le ministère de la Justice refuse d'autoriser la plainte dans $15 \%$ des dossiers 
qui relèvent de la Loi sur la conservation et la mise en valeur de la faune. Pour les $85 \%$ de causes qui restent, $7 \%$ se terminent avec un acquittement.

Les procureurs de la Couronne préferent, en général, le droit criminel au droit statutaire qui fut ajouté à leur tâche, un peu malgré eux, en 1976. Quelques-uns, responsables régionaux ou provincial du secteur pénal, y consacrent beaucoup plus de temps que d'autres. C'est pourquoi il est peu utile de parler d'une moyenne de temps investi dans ce secteur, en comparaison avec les causes criminelles. Par contre, à l'intérieur du secteur pénal, il est possible de tirer une conclusion du fait que tous ont affirmé que le nombre de causes reliées à la faune varie énormément d'une saison à l'autre. Les réponses des procureurs ont également démontré qu'il varie d'une région à l'autre, leurs estimations allant de $3 \%$, à Montréal, jusqu'à $95 \%$ dans des régions éloignées. Et bien que tous affirment que la majorité des causes pénales relèvent du Code de la route, ce sont les dossiers de la faune qui occupent, selon la région, une grande ou la majeure partie de leur temps dans ce secteur. La raison qu'ils en donnent est que ces procès sont spécialement longs et laborieux, lorsque procès il y a.

Pour les juges aussi, l'importance du secteur pénal dans leur charge de travail varie, entre $5 \%$ et $30 \%$. Le nombre de causes reliées à la faune qu'ils sont appelés à entendre fluctue. Lorsqu'ils tentent d'estimer la proportion du temps, à l'intérieur du secteur pénal, qu'ils consacrent à ces causes, la plupart en sont incapables et se contentent de souligner, selon les régions, que ces causes sont soit peu fréquentes, soit les deuxièmes en importance, après celles relevant du Code de la route.

Les juges et les procureurs n'évaluent l'importance des causes reliées à la faune pas seulement quantitativement, en termes de temps ou de nombre de causes. Leur perception dépend de leur sensibilité pour la faune et du fait qu'ils soient chasseurs et/ou pêcheurs ou non. Ils affirment que ces mêmes facteurs expliquent aussi certaines disparités qui existent, tant dans le traitement des causes que dans la sévérité des sentences demandées ou prononcées.

Procureurs de la Couronne et juges ont une image très bienveillante des agents de conservation de la faune, dont ils louent la motivation, le courage qu'ils démontrent dans un contexte de travail souvent difficile. Ils souhaiteraient les savoir mieux protégés. Ils reconnaissent leur savoir spécifique, bien qu'ils critiquent certaines carences dans leurs connaissances juridiques. Les critiques qu'ils expriment à l'égard des agents de conservation de la faune s'adressent pourtant plutôt au passé, puisque des 
cours de formation donnés récemment par un groupe de procureurs ont amené beaucoup d'améliorations. Ainsi, les dossiers sont maintenant mieux constitués et respectent un certain modèle. Les abus de pouvoir, surtout dans le domaine des saisies, se présentent moins souvent, et les droits de l'inculpé seraient mieux respectés. Ces améliorations récentes, les procureurs en assument volontiers la responsabilité, tout en soulignant aussi, qu'il y a moins d'acquittements depuis. Cependant, la plupart des interviewés insistent sur l'importance de la communication entre agents et procureurs en région et questionnent la directive de la part du ministère du Loisir, de la Chasse et de la Pêche, selon laquelle les agents devraient plutôt contacter le contentieux du ministère. Ils croient également qu'une meilleure gestion des ressources même restreintes permettrait aux agents d'enquêter d'une manière plus efficace.

Pour les juges et procureurs de la Couronne, le ministère du Loisir, de la Chasse et de la Pêche a une structure spéciale. Il est le seul ministère qui doit appliquer une législation dans le secteur pénal et qui possède son propre corps d'enquêteurs, les agents de conservation de la faune, qui sont des agents de paix, sous son contrôle. Cette dimension rend les causes qui relève de ce ministère comparables aux causes criminelles.

La plupart des interviewés, dans les deux groupes, soulignent un problème de communication avec le ministère. Ainsi, les juges souhaiteraient être consultés avant que le ministère ne procède à des modifications majeures de sa législation. Les procureurs se plaignent que le ministère ne les informe pas, parfois malgré des demandes répétées, sur la faune en général, son importance économique et écologique pour le Québec et les coûts causés par le braconnage. De telles informations seraient essentielles pour eux lorsqu'ils plaident. Ils demandent aussi que le MLCP leur fournisse des cartes et, à temps, les textes des dispositions législatives après chaque modification. Les juges se joignent à cette dernière demande, considérant qu'ils sont toujours les derniers servis.

Certaines politiques du ministère n'ont pas trouvé l'approbation des juges. Bien qu'ils ne mettent pas en doute le principe de l'accessibilité à la chasse, ils considèrent que la fermeture des clubs privés fut une erreur qui a coûté cher, puisqu'elle n'a pas été accompagnée d'une politique d'exploitation de la faune appropriée. Le contrôle sur les zones d'exploitation contrôlée (ZEC) mais également sur les parcs et les réserves fauniques, devrait être augmenté. Un juge propose même un retour en arrière, la transformation des ZEC en clubs privés. Les procureurs ne croient pas que le ministère ait une politique claire de répression du braconnage, sauf si l'on prend comme seul indicateur la sévérité des 
sanctions que la loi prévoit. Ainsi, le ministère du Loisir, de la Chasse et de la Pêche a longtemps hésité à investir dans la formation de ses agents. Il devrait miser sur les résultats plutôt que de limiter d'une manière rigide les ressources. Juges et procureurs critiquent aussi l'absence d'une politique de suivi des dossiers au ministère à Québec. L'information sur les antécédents des inculpés serait pourtant essentielle si l'on ne veut vider la notion de récidive de son sens, lorsque les sanctions alourdies que prévoit la loi en cas de récidive ne peuvent être appliquées, faute d'indications dans le dossier. Sur un autre plan, quelques procureurs soulignent que le système de permis, tel qu'il existe par exemple pour la chasse à l'orignal, crée des infractions techniques puisqu'il incite les chasseurs à le contourner. La raison de son maintien leur semble donc être plutôt une certaine rentabilité.

Les juges et les procureurs de la Couronne pensent par ailleurs que le ministère du Loisir, de la Chasse et de la Pêche s"intéresse plus à émettre des baux pour certains territoires qu'aux infractions, c'est pourquoi le contentieux du ministère n'aurait pas été à la hauteur de sa tâche, pendant une certaine période. Ils admettent cependant que la qualité des dossiers s'est améliorée entre-temps, surtout depuis que les plaintes sont autorisées par la direction des affaires pénales du ministère de la Justice. Les deux groupes critiquent la centralisation des plaintes au Service des infractions à Québec, d'une part parce que le phénomène du braconnage a ses particularités régionales dont il faut pouvoir tenir compte, d'autre part, parce que ce système lourd qui fait passer les dossiers entre plusieurs mains crée des délais importants. Lorsque le dossier arrive à la fin de son parcours sur le bureau du procureur qui doit le plaider, il est souvent trop tard pour demander un complément d'enquête, la prescription menace. Devant ce problème, ils proposent plusieurs solutions : le traitement local des dossiers, par le procureur en région, ou la nomination d'un procureur spécialisé qui sillonnerait la province. Ils sont pourtant conscients qu'une décentralisation entraînerait une surcharge de travail. Mais le contentieux du ministère n'est pas considéré comme seul responsable de ces délais importants; plusieurs les attribuent aux agents de conservation de la faune.

Tant les juges que les procureurs de la Couronne ont apprécié l'occasion qui leur a été donnée de se prononcer sur la législation sur la conservation et la mise en valeur de la faune. Dans les deux groupes, quelques interviewés ont exprimé leur satisfaction avec la législation actuellement en vigueur et sa tendance vers une plus grande sévérité. La majorité, par contre, ont exprimé des critiques, principalement sur les modifications fréquentes, le trop grand volume et le manque de clarté. 
Si quelques-uns critiquent ouvertement les modifications fréquentes qui entament le principe de la certitude de la loi, sèment la confusion chez les acteurs de l'appareil judiciaire et le public et, surtout, ne suivent aucune politique constante, d'autres les considèrent comme le signe d'une législation dynamique qui s'adapte à de nouveaux besoins. Cependant, la plupart des interviewés croient que l'ensemble du système judiciaire perd de sa crédibilité, lorsque ses acteurs ne se retrouvent pas dans la législation. Ils se sentent manipulés par les changements fréquents et recommandent de veiller à une meilleure application, plutôt que de modifier trop souvent les dispositions législatives.

Dans le même sens, plusieurs sont mécontents du trop grand nombre de dispositions législatives, devant lequel ils avouent démissionner, en ne vérifiant plus eux-mêmes.

Les procureurs de la Couronne ont appris avec satisfaction que la présomption de chasse de nuit ait été réintroduite, lors des dernières modifications, puisqu'ils pensent que l'on avait enlevé des instruments de travail importants aux agents. Sur un autre plan, ils considèrent que la législation ne tient pas assez compte des variations et spécificités régionales de la faune et du phénomène de braconnage. Comme solution, ils proposent la création d'une loi générale, accompagnée de règlements régionaux. Ils regrettent également que les deux niveaux législatifs, fédéral et provincial, ne suivent pas la même philosophie pénale dans le domaine de la faune ce qui entraîne des disparités dans les sentences.

Les juges constatent que la législation n'a pas assez d'impact et que son application manque d'efficacité. Ils se sentent par ailleurs limités dans leur pouvoir discrétionnaire, les amendes minimales leur laissant aucune marge de manœeuvre.

La plupart des causes dans lesquelles les agents enquêtent sont relativement simples et les infractions de nature plutôt technique. Le plus souvent, ce sont des informateurs qui attirent leur attention sur une possible infraction. L'informateur peut être une personne attitrée avec une certaine crédibilité qui sympathise avec les agents parce qu'elle est contre le braconnage ou, souvent, un voisin ou même un parent jaloux du succès du braconnier. Moins souvent, les agents découvrent eux-mêmes une infraction lors d'une patrouille. Exceptionnellement, les agents mènent de grandes opérations par lesquelles ils tentent de démanteler des réseaux de braconniers. Dans ces enquêtes qui impliquent souvent des agents d'autres régions, inconnus des braconniers, ils font de la surveillance intensive ou utilisent la technique de l'infiltration. 
En tant qu'agent de la paix, les agents de conservation de la faune ont des pouvoirs de saisie, de perquisition et d'arrestation. Lorsqu'ils constatent une infraction, ils saisissent en général les animaux ou poissons chassés ou capturés illégalement et les instruments ayant servi à commettre l'acte, surtout les armes. Juges et procureurs constatent avec satisfaction que les agents ont tendance à saisir moins, depuis quelques années, et qu'ils s'en abstiennent de plus en plus lorsqu'il s'agit d'une infraction technique. L'idéal serait, selon les procureurs, que les agents ne saisissent que ce qui sera matière à confiscation. Ils devraient donc tenir compte des délais à respecter et de la gravité de l'infraction, en fonction de laquelle le juge prononcera la confiscation. Les juges approuvent que les confiscations obligatoires qui leur enlevaient leur pouvoir discrétionnaire disparaissent en partie, avec les dernières modifications apportées à la loi.

Depuis récemment, la présentation des rapports d'enquête par les agents s'est grandement améliorée. Les procureurs reconnaissent qu'il y a un effort d'uniformiser la constitution des dossiers. Ils critiquent, par contre, le fait que la preuve reste souvent insuffisante, lorsque le dossier leur est acheminé. Et tandis que quelques procureurs en tiennent les agents responsables, d'autres l'imputent au contentieux du MLCP qui exercerait mal son contrôle. Dans les deux cas, ils suggèrent aux agents de communiquer avec eux à temps. Les juges et les procureurs constatent pourtant une amélioration, depuis que la direction des affaires pénales du ministère de la Justice autorise les plaintes et exerce un dernier contrôle.

Les procureurs rapportent que $80 \%$ à $90 \%$ des causes se règlent par un plaidoyer de culpabilité. Différents facteurs peuvent influencer le nombre de plaidoyers de culpabilité offerts: la qualité de l'enquête, le montant minimum de l'amende, le type d'infraction, la valeur des objets saisis qui risquent d'être confisqués, les frais associés au procès, surtout les frais de déplacements pour les témoins.

Il y a procès, si la plainte est contestée. L'issue du procès est, selon les données du ministère de Ja Justice pour l'année 1986, dans la plupart des causes une condamnation. Dans $7 \%$ des cas, le juge acquitte. Les procureurs de la Couronne soulignent l'importance de leur rôle pour l'issue du procès. Ils pensent également pouvoir l'influencer, en préparant le procès avec l'agent qui a mené l'enquête et en rencontrant les autres témoins appelés à comparaitre.

Les procès en matière de faune ressemblent aux causes criminelles. Juges et procureurs les comparent aux causes criminelles les plus difficiles et les plus longues. Tout y est contesté; les peines minimales étant 
fixées par la loi, il n'est pas question de faire des représentations sur sentences.

Généralement, la preuve est circonstancielle, une preuve directe étant rare. Elle s'appuie principalement sur les témoignages des agents de conservation de la faune auxquels les juges reprochent d'exagérer ou de faire des luttes personnelles de leurs causes. Or, dans ce petit milieu, la crédibilité entamée d'un agent est difficilement réparable. Plusieurs procureurs ont pourtant constaté, depuis peu, une amélioration dans la façon de témoigner des agents. Des témoins civils ne sont que rarement entendus. Les procureurs considèrent par ailleurs une erreur stratégique de faire témoigner les délateurs, tant parce que leur témoignage n'est plus nécessaire, dès que les agents ont constaté eux-mêmes l'événement, qu'à cause du fait que les juges ne leur accordent que peu de crédibilité. Le temps est un autre facteur qui risque de provoquer l'acquittement, faisant oublier aux témoins des détails importants.

Quelques juges regrettent que les accusés ne se font pas assez souvent défendre dans les procès en matière statutaire, bien qu'ils soulignent qu'ils le font quand même plus dans les causes liées à la faune qu'ailleurs. Les défenses qu'ils avancent leur paraissent souvent farfelues.

Juges et procureurs constatent des disparités dans le comportement décisionnel des juges qui se réflètent plus dans le traitement des causes que dans la sévérité des sentences. Pour les procureurs, la notion du doute raisonnable semble être plus élastique dans l'attitude des juges lorsqu'ils décident d'une cause pénale que d'une cause criminelle. C'est pourquoi ils acquittent plus facilement. Rappelons que le braconnage relève du droit pénal provincial. Mais l'issue d'un procès dépend aussi de leur intérêt pour la faune. Les procureurs de la Couronne affirment pourtant pouvoir influencer l'attitude des juges.

Les sentences prononcées correspondent habituellement au minimum fixé dans la loi. Le juge suit les représentations du procureur, lequel, de son côté, tient compte des suggestions de l'agent de conservation de la faune qui a mené l'enquête.

La législation sur la conservation et la mise en valeur de la faune comporte des peines minimales obligatoires, un instrument de dissuasion rarement utilisé en droit pénal canadien. Au niveau fédéral, toutes les commissions ${ }^{5}$ qui, depuis 35 ans, se sont penchées sur la question ont

5. La Commission royale de la révision du Code criminel (1952); le Comité Ouimet (1969); la Commission de la réforme du droit du Canada (1975); la Commission canadienne sur la détermination de la peine (1987). 
recommandé leur abolition. Les recherches ont démontré que les peines minimales obligatoires n'ont aucun effet dissuasif spécial, le public n'étant même pas au courant, tant de leur seuil que de leur existence. Il est établi qu'elles tendent à corrompre l'administration de la justice qui trouve des moyens de les contourner. Ainsi, l'argument principal avancé en leur faveur, c.-à-d. l'uniformité des sentences, est mis en doute. L'analyse du discours des juges et des procureurs de la Couronne confirme que le débat perdure. Quelques-uns considèrent que des peines minimales obligatoires constituent un affront à l'endroit des juges, un manque de confiance, comme s'ils ne savaient pas évaluer une infraction dans son contexte. Pour d'autres, elles empêchent le juge de tenir compte des critères objectifs et subjectifs. Plusieurs pensent aussi que le seuil minimal est trop haut, tandis que certains attribuent une telle attitude à des juges peu sensibilisés à la faune. Pourtant, la majorité des interviewés trouvent les amendes disproportionnées lorsqu'ils les comparent aux peines imposée dans d'autres causes, relevant d'autres lois provinciales ou fédérales, entendues dans la même journée. Quelques procureurs, souhaitant la réalisation des principes d'équité et d'uniformité des sentences, suggèrent plutôt que des peines minimales obligatoires des lignes directrices pour la détermination de la peine, comme elles existent dans certains États américains.

Les récentes augmentations des peines apportées à la Loi sur la conservation et la mise en valeur de la faune provoquent des réactions partagées tant chez les juges que chez les procureurs. Quelques juges s'opposent et recommandent de voir plutôt à une meilleure application de la loi et au respect des sentences prononcées. Pour la plupart, l'acceptabilité des augmentations est intimement liée à leur attitude face aux peines minimales obligatoires. Si la majorité des procureurs est contre une augmentation des peines minimales, considérant que la plupart des condamnés ne réussissent pas à payer les amendes actuellement imposées, ils s'opposent moins dans le cas du récidiviste notoire. Ils sont, par ailleurs, convaincus que des peines minimales obligatoires plus élevées provoqueraient un plus grand nombre d'acquittements et une diminution du nombre de plaidoyers de culpabilité. Plusieurs soulignent aussi la disparité flagrante qu'ils constatent, en comparaison avec d'autres causes. Même avant les récentes modifications, les amendes étaient beaucoup plus élevées que celles imposées par les juges dans des causes de vol, de conduite avec facultés affaiblies, etc.

Les attitudes des juges et des procureurs envers la peine d'incarcération pour les braconniers varient. Inposée ou non, elle n'est de toutes façons pas respectée, à la fois parce que les prisons sont pleines et parce 
qu'elle coûte cher. Les interviewés affirment que les condamnés en fonction de dispositions statutaires sont les derniers à purger leur sentence, l'échelle de gravité les plaçant en dernier. Il y a pourtant des défenseurs de l'incarcération de braconniers condamnés, aussi bien parmi les juges que les procureurs de la Couronne. Ils croient que l'incarcération reste inévitable dans des cas extrêmes d'individus qui ne comprennent pas, qui sont "comme des enfants". D'autres indiquent que l'incarcération peut constituer un moyen pour contourner des amendes minimales trop lourdes.

Tous les répondants ont affirmé que la sentence prononcée habituellement, dans le contexte de peines minimales obligatoires, est le minimum prévu. Aucun n'avait connaissance d'une cause où le maximum prévu aurait été imposé. Afin de prononcer une sentence qui dépasse la peine minimale, des facteurs aggravants doivent être présents. Il s'agit soit de moyens employés spécialement répugnants, de braconnage en réseaux bien structurés ou, selon un juge, de touristes américains qu'il fallait dissuader. Mais même si l'infraction est considérée comme spécialement grave, juges et procureurs de la Couronne ne sont pas nécessairement prêts à dépasser la peine minimale obligatoire. Ils la considèrent assez élevée et rappellent que la condamnation a également d'autres conséquences importantes. La confiscation d'objets saisis peut souvent frapper plus que l'amende, surtout lorsqu'il s'agit d'armes ou de lignes de pêche. En plus, le condamné assume les frais, souvent élevés, lorsque des témoins ont dû se déplacer sur des distances importantes. Finalement, une condamnation est en général accompagnée de peines administratives, comme le retrait du permis de chasse, etc. Dans ce contexte, un procureur recommande de suivre le modèle du Code criminel et d'interdire la possession d'armes pour une certaine période, dans des cas de braconnage grave. Par ailleurs, l'importance des confiscations se reflète également dans le fait qu'elles font souvent l'objet de négociations entre procureur de la Couronne et la défense. Et tandis que les juges s'opposent aux confiscations obligatoires qui annulent leur pouvoir discrêtionnaire, les procureurs les approuvent puisqu'elles réduiraient les disparités entre juges.

Ce n'est que dans le domaine de la pêche que des barèmes de sentences ont été établis, dans différentes régions. Lorsque la loi prévoit des peines minimales obligatoires, la question ne se pose pas. L'utilité de créer des barèmes semble par ailleurs liée au nombre de causes qui se présentent dans une région. Certains s'y opposent par principe, insistant qu'il faut juger l'acte plutôt que la quantité. Des barèmes sont forgés soit par les juges d'une région, soit par les procureurs, soit suite à des ententes 
entre ces deux groupes, soit encore par les agents de conservation qui utilisent alors le procureur comme intermédiaire. Ils pourraient également l'être par le ministère du Loisir, de la Chasse et de la Pêche ou par le ministère de la Justice, ce qui ne semble pas être le cas actuellement. Finalement, plusieurs juges et procureurs se sont indignés devant le fait que les sentences imposées ne soient guère respectées.

Juges et procureurs de la Couronne favoriseraient une plus grande publicité des causes de braconnage dans les médias, surtout dans les périodes précédant l'ouverture de la chasse, bien qu'ils voient la priorité dans une sensibilisation positive du public.

Notre analyse du phénomène braconnage et de l'application de la législation sur la conservation et la mise en valeur de la faune, à partir des perceptions et pratiques des juges et des procureurs de la Couronne, a identifié des points faibles de ce sous-système de l'administration de la justice. D'importance inégale, ils sont pourtant tous liés, d'une manière ou d'une autre, au constat global qui s'impose : la communication, tant à l'intérieur qu'à l'extérieur du système, présente des failles. Bien qu'il y ait eu amélioration, depuis deux ans, à plusieurs égards, des ambiguités demeurent sur les enjeux du contrôle du braconnage et les moyens de conservation de la faune. Cette note de recherche ne peut tout dire. Pourtant, les points suivants mériteraient réflexions et élaborations: la notion de braconnage devrait être qualifiée et les peines minimales obligatoires repensées devant l'évidence qu'elles n'atteignent pas l'effet dissuasif escompté, tout en alourdissant l'administration de la justice. L'efficacité sociale et juridique de la loi sur la conservation et la mise en valeur de la faune serait mieux servie par des actions concertées entre les appareils législatifs et administratifs et une meilleure éducation du public. 